\title{
Adipose tissue is required for the antidiabetic, but not for the hypolipidemic, effect of thiazolidinediones
}

\author{
Lily Chao, ${ }^{1}$ Bernice Marcus-Samuels, ${ }^{1}$ Mark M. Mason, ${ }^{1}$ Jaideep Moitra, ${ }^{2}$ \\ Charles Vinson, ${ }^{2}$ Elif Arioglu, ${ }^{1}$ Oksana Gavrilova, ${ }^{1}$ and Marc L. Reitman ${ }^{1}$ \\ ${ }^{1}$ Diabetes Branch, National Institute of Diabetes and Digestive and Kidney Diseases, NIH, Bethesda, Maryland, USA \\ ${ }^{2}$ Laboratory of Biochemistry, National Cancer Institute, NIH, Bethesda, Maryland, USA \\ Address correspondence to: Marc Reitman, Building 10, Room 8N-250, Diabetes Branch, \\ National Institute of Diabetes and Digestive and Kidney Diseases, NIH, Bethesda, Maryland 20892-1770, USA. \\ Phone: (301) 496-6090; Fax: (301) 402-5788; E-mail: marc.reitman@nih.gov. \\ Received for publication September 6, 2000, and accepted in revised form October 11, 2000.
}

\begin{abstract}
There is uncertainty about the site(s) of action of the antidiabetic thiazolidinediones (TZDs). These drugs are agonist ligands of the transcription factor PPAR $\gamma$, which is abundant in adipose tissue but is normally present at very low levels in liver and muscle. We have studied the effects of TZDs in AZIP/F-1 mice, which lack white adipose tissue. The A-ZIP/F-1 phenotype strikingly resembles that of humans with severe lipoatrophic diabetes, including the lack of fat, marked insulin resistance and hyperglycemia, hyperlipidemia, and fatty liver. Rosiglitazone or troglitazone treatment did not reduce glucose or insulin levels, suggesting that white adipose tissue is required for the antidiabetic effects of TZDs. However, TZD treatment was effective in lowering circulating triglycerides and increasing whole body fatty acid oxidation in the A-ZIP/F-1 mice, indicating that this effect occurs via targets other than white adipose tissue. A-ZIP/F-1 mice have markedly increased liver PPAR $\gamma$ mRNA levels, which may be a general property of fatty livers. Rosiglitazone treatment increased the triglyceride content of the steatotic livers of A-ZIP/F-1 and $o b / o b$ mice, but not the "lean" livers of fat-transplanted A-ZIP/F-1 mice. In light of this evidence that rosiglitazone acts differently in steatotic livers, the effects of rosiglitazone, particularly on hepatic triglyceride levels, should be examined in humans with hepatic steatosis.
\end{abstract}

J. Clin. Invest. 106:1221-1228 (2000).

\section{Introduction}

Diabetes mellitus is a disease defined by elevated blood glucose levels. The causes of type 2 diabetes are not well understood. It is thought that both resistance of target tissues to the action of insulin and decreased insulin secretion (" $\beta$-cell failure") occur. Major insulinresponsive tissues for glucose homeostasis are liver, in which insulin stimulates glycogen synthesis and inhibits gluconeogenesis; muscle, in which insulin stimulates glucose uptake and glycogen synthesis; and white adipose tissue (WAT), in which insulin stimulates glucose uptake and inhibits lipolysis. The relative importance of each tissue is not known; nor are the details of how they interact.

To understand the physiological roles of adipose tissue, we have generated a transgenic line of mice, named A-ZIP/F-1, which has virtually no white adipose tissue (1). These mice express a dominant negative protein in adipose tissue that heterodimerizes with and inactivates members of the C/EBP and JUN families of B-ZIP transcription factors. The A-ZIP/F-1 phenotype strikingly resembles that of humans with severe lipoatrophic diabetes, including the lack of fat, marked insulin resistance and hyperglycemia, hyperlipidemia, fatty liver, and organomegaly. The detailed mechanisms by which the lack of WAT causes insulin resistance are not established. Contributing components probably include deficiency of leptin (and other hormones) and the lack of a place to store triglycerides $(2-5)$. It has been postulated that increased intracellular triglycerides (or metabolites such as fatty acyl-CoA or malonyl-CoA) inside nonadipose tissues could cause insulin resistance $(6,7)$.

The TZDs are a class of antidiabetic agents that act by increasing insulin sensitivity $(8,9)$. Currently, two drugs in this class are approved for use in the United States, rosiglitazone (formerly BRL49653; ref. 10) and pioglitazone. TZDs are agonist ligands for the transcription factor peroxisome proliferator-activated receptor $\gamma$ (PPAR $\gamma$ ), and their antidiabetic effects are thought to be mediated through PPAR $\gamma(11)$. PPAR $\gamma$ is expressed at high levels in adipose tissue, colon, and activated macrophages, and at lower levels in other tissues (12-17). The tissues at which TZDs act to increase insulin sensitivity are debated $(8,9,18)$. TZDs increase glucose utilization (largely a muscle function) and, at higher doses, inhibit endogenous glucose production (largely a liver function) (19). However, liver and muscle have low PPAR $\gamma$ levels. Thus, WAT has been a leading candidate for the target of TZD actions, as it is the only insulin-responsive tissue with a high level of PPAR $\gamma$. Additionally, PPAR $\gamma$ and its agonist ligands stimulate 
adipose differentiation (20), leading to increased numbers of small adipocytes, which are thought to be more insulin sensitive than are large, lipid-laden adipocytes (21). However, Burant et al., studying a partially lipoatrophic mouse, concluded that troglitazone effectively treated diabetes, independent of adipose tissue (22). Here, we have reexamined this question using the more severely lipoatrophic A-ZIP/F-1 mice and do not see an effect of TZDs on insulin and glucose levels. However, rosiglitazone greatly increases the hepatic steatosis of these mice, which may be due to their increased hepatic expression of PPAR $\gamma$.

\section{Methods}

Mice. Generation and characterization of the "fatless" A-ZIP/F-1 mice have been reported $(1,3-5,23)$. Studies were approved by the NIDDK animal care and use committee. All A-ZIP/F-1 mice used in this study were hemizygous females on the FVB/N background, produced by breeding hemizygous males with wild-type (WT) females. Controls were age- and sex-matched FVB/N mice. C57BL/6J-lep ${ }^{o b / o b}$ female mice were purchased from The Jackson Laboratory (Bar Harbor, Maine, USA). Mice were typically reared three to a cage on a 12hour light/dark cycle (lights on at 0600-1800 hours), fed water and pellet (NIH-07) or powder (AIN 93G; Dyets, Bethlehem, Pennsylvania, USA) diet ad libitum. Food was blended daily with rosiglitazone $(0.012 \mathrm{mg} / \mathrm{g}$ diet, for a dose of $\sim 3 \mathrm{mg} / \mathrm{kg} / \mathrm{d}$; Smith Kline Beecham, West Sussex, United Kingdom) in a coffee grinder. Troglitazone (Parke-Davis, Ann Arbor, Michigan, USA) treatment was given by oral gavage at dose of 10 $\mathrm{mg} / 100 \mu \mathrm{l} \mathrm{H}_{2} \mathrm{O}$ daily for 2 weeks. All mice were sacrificed at between 0900 and 1200 hours. Tissues were fixed in neutralized $10 \%$ formalin and then processed by American Histolabs (Gaithersburg, Maryland, USA).

RNA analysis. cDNA probes for mouse acyl-CoA oxidase (AOX) and PPAR $\alpha$ (courtesy of J. Peters, National Cancer Institute; ref. 24) and phosphoenolpyruvate carboxykinase (PEPCK) (25) were excised from plasmids. cDNA probes for sterol regulatory element binding protein-1 (SREBP1), stearoyl-CoA desaturase-1 (SCD1), and fatty acid synthase (FAS) were prepared by PCR (26) with mouse liver cDNA. PCR, using mouse liver cDNA as template with the following primers, was used to make probes for carnitine palmitoyl transferase I (CPT1: 5' primer, 5'GAGAGCTGGCAACACCATCCACGC3'; 3'-primer, 5'GGGATtTGgCGTAGCTGTCGATGG $\left.3^{\prime}\right)$, PPAR $\gamma \quad\left(5^{\prime}\right.$ primer, 5'ACAAGACTACCCTTTACTGAAATTACCATG3'; 3'primer, 5'GGCACTTCTGAAACCGACAGTACTGACATTTATTT3'), and insulin receptor substrate-2 (IRS2: 5'-primer, 5'GCGGCCTCATCTTCTTCACTGC3'; 3'-primer, 5'GGTTCCACGgCCTAGCCTGTGG3'). Total RNA was extracted using TRIzol (Life Technology Inc., Rockville, Maryland, USA). Northern blots (on Maximum Strength Nytran Plus; Schleicher \& Schuell, Keene, New Hampshire, USA) were hybridized using Rapid-hyb (Amersham Pharmacia, Piscataway, New Jersey, USA) according to the manufacturer's instructions and exposed to film or quan- titated with a BioImaging Analyzer with BAS 1500 MacBus software (Fuji Medical Systems, Standish, Maine, USA). Background was corrected by subtracting the average of an adjacent area on the blot.

Biochemical assays. FFAs, triglycerides, and insulin were assayed as described elsewhere (4). Glycated hemoglobin was assayed using a kit (442-B; Sigma Chemical Co., St. Louis, Missouri, USA). Glucose was measured using a Glucometer Elite (Bayer Corp., Elkhart, Indiana, USA), and serum chemistries were measured by the Clinical Pathology Department of the $\mathrm{NIH}$ Clinical Center. Liver triglyceride was measured by ethanolic $\mathrm{KOH}$ hydrolysis followed by a radiometric assay for glycerol (23).

Indirect calorimetry. Oxygen consumption and carbon dioxide production were measured as described previously (4). Measurements were made simultaneously with mice from different groups. In fasting animals, food was removed at onset of recording; data points from the 4th to 7th hours inside the metabolic chamber were used for analysis. Resting oxygen consumption was calculated as the average of the points with less than 21 ambulating beam breaks per minute.

Fat transplantation. Five-week-old A-ZIP/F-1 females $(\sim 17 \mathrm{~g})$ were anesthetized with pentobarbital $(0.01 \mathrm{ml} / \mathrm{g}$ body weight of $5 \mathrm{mg} / \mathrm{ml}$ in $5 \%$ ethanol/PBS, intraperitoneally). Donor fat pads (parametrial) from agematched WT female mice sacrificed by cervical dislocation were placed into sterile $0.9 \%$ saline. Four fat pads were implanted subcutaneously through small incisions in the shaved skin of the back, with one piece per incision (4). Silk sutures (4-0) were used to close incisions. Mice were housed singly for 3 days after surgery and then at three to a cage. Tail venous blood $(\sim 150 \mu \mathrm{l})$ was withdrawn at noon 1 day before surgery, and weekly thereafter. Upon sacrifice, the grafts were examined visually, weighed, and fixed for histological analysis.

Statistical analysis. Values are reported as means \pm SEM. Statistical significance was determined using twotailed $t$ test or ANOVA, as appropriate.

\section{Results}

Rosiglitazone does not improve the diabetes of A-ZIP mice. Treatment of A-ZIP/F-1 mice for 5 weeks with rosiglitazone had no effect on their threefold elevated glucose levels or the 200-fold elevated insulin levels (Figure 1). Similarly, treatment for 2 weeks with troglitazone failed to reduce the glucose and insulin levels (Table 1). As a positive control for TZD effectiveness, we treated leptindeficient $o b / o b$ mice with rosiglitazone (Figure 1). As expected (27), the $o b / o b$ mice showed vastly improved insulin levels and normalization of serum glucose.

A glucose tolerance test was performed to characterize the insulin resistance further. In the A-ZIP/F-1 mice, the baseline 6-hour-fasted blood glucose values were elevated, and the glucose levels rose higher and remained elevated for longer than in WT controls (Figure 2). Rosiglitazone treatment of the A-ZIP/F-1 mice did not alter the abnormal glucose tolerance test. Thus, 
in the lipoatrophic and diabetic A-ZIP/F-1 mice, rosiglitazone did not ameliorate the insulin resistance, confirming that adipose tissue is required for the glucose- and insulin-lowering effects of TZDs.

Rosiglitazone improves the lipid profile of A-ZIP/F-1 mice. In addition to their insulin-sensitizing actions, TZDs lower serum triglycerides and FFAs $(22,28-30)$. In marked contrast to its lack of effect on glucose and insulin levels, rosiglitazone treatment lowered serum triglyceride and FFA levels to normal in the A-ZIP/F-1 mice (Figure 1). Troglitazone was also effective at lowering the triglycerides and FFA levels (Table 1), suggesting that this is a general property of TZDs. Taken together, these data demonstrate that TZDs can reduce serum triglyceride and FFA levels in mice lacking adipose tissue.

Rosiglitazone exacerbates hepatic steatosis of A-ZIP/F-1 mice. We next examined the mechanism by which TZDs lower triglycerides and FFAs in lipoatrophic mice. Improvement of serum triglycerides and FFAs could be caused by redistribution from the circulation to tissue sites. Because A-ZIP/F-1 mice lack WAT, increased lipid deposition in this tissue cannot occur. However, the liver can store prodigious amounts of triglyceride. At baseline, A-ZIP/F-1 livers are already very steatotic (1, $5,23)$. However, the livers from rosiglitazone-treated mice were even larger (1.87-fold) and lighter in color than the untreated A-ZIP/F-1 livers (Figure 3; Table 2). Histologically, the rosiglitazone-treated livers showed increased vacuolation, indicative of lipid deposition. Rosiglitazone treatment had no effect on the gross appearance or histology of WT livers.

Next, hepatic triglyceride content was measured directly. Rosiglitazone caused a 3.5-fold increase in liver triglyceride concentration in the A-ZIP/F-1 mice and a 1.4-fold increase in the $o b / o b$ mice, compared with a $35 \%$ reduction in WT mice (Figure $3 i$ ). When triglyceride content was expressed as a total per liver, the effect of rosiglitazone was even more remarkable (AZIP/F-1, $432 \pm 125$ vs. $2,781 \pm 414 \mu \mathrm{mol}, P<0.001$; $o b / o b, 1,497 \pm 172$ vs. $2,516 \pm 744 \mu \mathrm{mol}, P=0.01$; WT, $54 \pm 7$ vs. $31 \pm 4 \mu \mathrm{mol}, P=0.02$; in each pair, values are for control and rosiglitazone-treated, respectively). In contrast to rosiglitazone, troglitazone treatment did
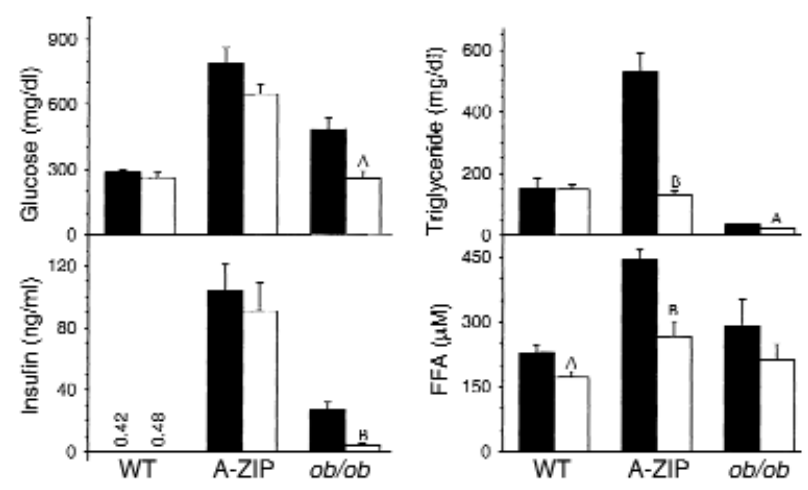

\section{Figure 1}

Serum chemistries of nonfasted female mice. WT or A-ZIP/F-1 mice, 4-5 weeks old, were treated with a control (filled bars) or rosiglitazone (open bars) diet for 5 weeks. Female $o b / o b$ mice, 6 weeks old, were treated for 2 weeks. Data are mean $\pm \operatorname{SEM}(n=6)$. ${ }^{A} P<0.05$ for differences within each genotype between control and rosiglitazonetreated mice. ${ }^{\mathrm{B}} P<0.005$ for differences within each genotype between control and rosiglitazone-treated mice.

not increase liver triglyceride in A-ZIP/F-1 mice (Table 1). Similar to the effect of rosiglitazone, troglitazone treatment of control mice reduced liver triglycerides.

During rosiglitazone treatment, the A-ZIP/F-1 mice gained weight, which was quantitatively accounted for by the increase in liver size (Table 2). Rosiglitazone also caused a significant, but less dramatic increase in liver weight in the ob/ob mice. By comparison, rosiglitazone treatment of the WT mice caused no change in body weight and a small reduction in liver weight.

Given the marked steatosis and hepatomegaly (to 18\% of body weight), we looked for evidence of altered liver function or hepatitis. Serum biochemistries in rosiglitazone-treated A-ZIP/F-1 mice showed unchanged albumin and slight increases in transaminases that were not statistically significant (Table 2). Serum total bilirubin, lipase, and cholesterol, on the other hand, decreased significantly. In addition, there was no histological evidence of hepatitis. These data suggest that the liver is functioning normally, with little evidence for liver injury, despite the massive steatosis.

Table 1

Treatment of A-ZIP/F-1 mice with troglitazone

\begin{tabular}{lcccc}
\hline & $\begin{array}{c}\text { WT, } \\
\text { Control }\end{array}$ & $\begin{array}{c}\text { WT, } \\
\text { Troglitazone }\end{array}$ & $\begin{array}{c}\text { A-ZIP/F-1, } \\
\text { Control }\end{array}$ & $\begin{array}{c}\text { A-ZIP/F-1, } \\
\text { Troglitazone }\end{array}$ \\
Body weight $(\mathrm{g})$ & $22.8 \pm 0.8$ & $22.3 \pm 0.8$ & $23.9 \pm 1.3$ & $23.7 \pm 0.9$ \\
Food intake $(\mathrm{g} / \mathrm{d})$ & $3.92 \pm 0.18$ & $3.83 \pm 0.16$ & $5.33 \pm 0.18$ & $4.91 \pm 0.28$ \\
Liver weight $(\mathrm{g})$ & $1.07 \pm 0.02$ & $1.06 \pm 0.03$ & $2.60 \pm 0.26$ & $67 \pm 5$ \\
BAT weight $(\mathrm{mg})$ & $120 \pm 13$ & $144 \pm 3$ & $929 \pm 90$ & $53 \pm 6$ \\
Glucose $(\mathrm{mg} / \mathrm{dl})$ & $294 \pm 18$ & $271 \pm 23$ & $113 \pm 24$ & $859 \pm 58$ \\
Insulin $(\mathrm{ng} / \mathrm{ml})$ & $0.93 \pm 0.20$ & $262 \pm 0.08$ & $928 \pm 150$ & $99 \pm 31$ \\
FFA $(\mu \mathrm{M})$ & $312 \pm 43$ & $259 \pm 34$ & $919 \pm 83$ & $59 \pm \pm 58^{\mathrm{A}}$ \\
Triglyceride $(\mathrm{mg} / \mathrm{dl})$ & $300 \pm 43$ & $24 \pm 5^{\mathrm{A}}$ & $279 \pm 34$ & $281 \pm 21$ \\
Liver triglyceride $(\mu \mathrm{mol} / \mathrm{g})$ & $50 \pm 6$ & $25 \pm 5^{\mathrm{A}}$ & $732 \pm 116$ & $751 \pm 63$ \\
Liver triglyceride $(\mu \mathrm{mol} /$ liver $)$ & $53 \pm 7$ & & & \\
\hline
\end{tabular}

Female WT or A-ZIP/F-1 mice, 6-8 weeks old, were treated with troglitazone for 2 weeks. Animals were sacrificed in the fed state. Data are mean \pm SEM $(n=6) .{ }^{A} P<0.05$ for difference within genotype between control and troglitazone-treated mice. 


\section{Figure 2}

Glucose tolerance testing. Intraperitoneal glucose tolerance tests using 2 $\mathrm{mg}$ glucose/ $\mathrm{kg}$ were performed at approximately 8 hours after lights on, after a 6-hour fast (4). Female WT (circles) and A-ZIP/F-1 (triangles) mice were treated with rosiglitazone (open symbols) or control diets (filled symbols) for 2-4 weeks at the time of testing. Tail vein blood was collected immediately before and at 15, 30, 60, and 120 minutes after glucose injection. Data are the average of two tests per mouse, performed 1 week apart, with six mice per group. A-ZIP/F-1 mice are different from WT at $P<0.05$ for all points, with no effect of rosiglitazone treatment.

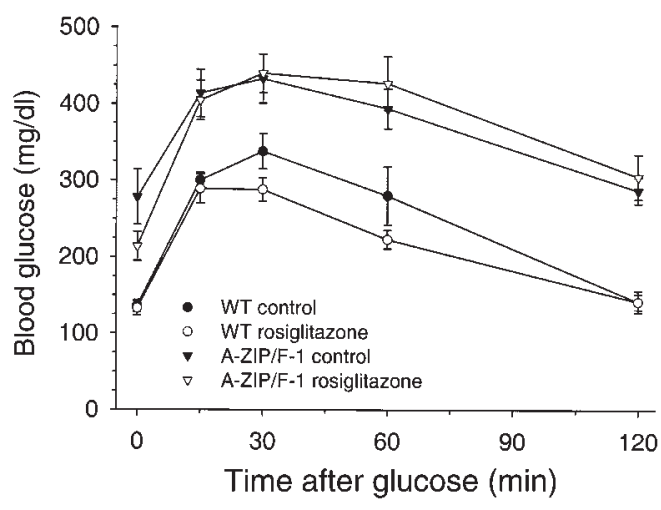

Rosiglitazone increases respiratory exchange ratio. The respiratory exchange ratio (RER; the ratio of $\mathrm{CO}_{2}$ produced to $\mathrm{O}_{2}$ consumed) is a measure of metabolic fuel source. Pure carbohydrate oxidation gives an RER of 1.00 , whereas pure fat oxidation gives a ratio of approximately 0.71 (31). Fasting lipoatrophic humans (32) and A-ZIP/F-1 mice (33) have an elevated RER, indicating increased net fractional oxidation of carbohydrate. In WT mice, 4-7 hours of fasting was sufficient to reduce the RER maximally, irrespective of treatment with rosiglitazone. Under identical conditions, the AZIP/F-1 mice did not show a maximally reduced RER unless treated with rosiglitazone (Table 2). Thus rosiglitazone increases net fractional lipid oxidation, suggesting that increased fat oxidation (or decreased lipogenesis) contributes to rosiglitazone's ability to reduce serum FFA and triglyceride levels.

Diabetes of fat-transplanted mice does not respond to rosiglitazone. We next studied whether the antidiabetic effect of rosiglitazone could be restored in A-ZIP/F-1 mice by transplantation of WT adipose tissue. A submaximal transplant amount (400 mg) (4) was chosen, enabling the potential for detection of a rosiglitazone effect. Similar to previous observations $(4,5)$, fat transplantation improved the glucose and insulin levels beginning
2 weeks after surgery, whereas sham-operated mice had persistent hyperglycemia and hyperinsulinemia (Figure 4 , $a$ and $b$ ). However, we did not detect any effect of rosiglitazone treatment on the final glycated hemoglobin (sham control, $11.6 \pm 1.1 \%$; sham rosiglitazone, 12.3 $\pm 0.8 \%$; transplanted control, $7.2 \pm 0.6 \%$; transplanted rosiglitazone, $7.8 \pm 1.2 \%$; WT $6.6 \pm 0.5 \%$ ), glucose, or insulin levels in the fat-transplanted mice (data not shown). The rosiglitazone treatment was effective, as the weight of the brown adipose tissue in both the sham- and fat-transplanted groups was increased by rosiglitazone treatment (sham, from $60 \pm 10$ to $177 \pm$ $21 \mathrm{mg}$; transplanted, from $27 \pm 3$ to $66 \pm 6 \mathrm{mg}$ ).

We next examined the effect of fat transplantation on the increased hepatic size and steatosis caused by rosiglitazone treatment (Figure 4, $\mathrm{c}$ and d). As expected $(4,5)$, fat transplantation greatly decreased liver weight (by $42 \%$ ) and triglyceride content (by $82 \%$ ), but did not reduce either to WT levels. However, rosiglitazone treatment did not increase hepatic triglyceride content; the steatotic effect of rosiglitazone was abolished by WAT transplantation.

Hepatic PPAR $\gamma$ expression correlates with rosiglitazoneinduced increase in liver triglyceride content. Given that hepatic levels of PPAR $\gamma$ are a possible determinant of

Table 2

Characteristics of rosiglitazone-treated mice

\begin{tabular}{|c|c|c|c|c|c|c|}
\hline & $\begin{array}{l}\text { WT, } \\
\text { Control }\end{array}$ & $\begin{array}{c}\text { WT, } \\
\text { Rosiglitazone }\end{array}$ & $\begin{array}{l}\text { A-ZIP/F-1, } \\
\text { Control }\end{array}$ & $\begin{array}{c}\text { A-ZIP/F-1, } \\
\text { Rosiglitazone }\end{array}$ & $\begin{array}{l}\text { ob/ob, } \\
\text { Control }\end{array}$ & $\begin{array}{c}\text { ob/ob, } \\
\text { Rosiglitazone }\end{array}$ \\
\hline Body weight (g) & $21.9 \pm 0.5$ & $22.1 \pm 0.4$ & $22.4 \pm 0.4$ & $24.2 \pm 0.4^{\mathrm{A}}$ & $39.3 \pm 0.6$ & $41.3 \pm 0.6^{A}$ \\
\hline Liver weight (g) & $0.95 \pm 0.02$ & $0.83 \pm 0.03^{\mathrm{A}}$ & $2.28 \pm 0.13$ & $4.27 \pm 0.31^{\mathrm{B}}$ & $3.69 \pm 0.11$ & $4.51 \pm 0.19^{\mathrm{B}}$ \\
\hline BAT weight $(\mathrm{mg})$ & $125 \pm 6$ & $271 \pm 14^{\mathrm{B}}$ & $65 \pm 12$ & $150 \pm 17^{B}$ & $340 \pm 12$ & $531 \pm 28^{A}$ \\
\hline Aspartate aminotransferase $(\mathrm{U} / \mathrm{I})$ & $158 \pm 18$ & $126 \pm 18$ & $211 \pm 57$ & $323 \pm 71$ & & \\
\hline Alanine aminotransferase $(\mathrm{U} / \mathrm{I})$ & $51 \pm 9$ & $44 \pm 7$ & $101 \pm 25$ & $189 \pm 47$ & & \\
\hline Lipase $(U / I)$ & $46 \pm 3$ & $47 \pm 8$ & $53 \pm 7$ & $35 \pm 4^{\mathrm{A}}$ & & \\
\hline Cholesterol (mg/dl) & $118 \pm 5$ & $119 \pm 4$ & $171 \pm 11$ & $134 \pm 12^{\mathrm{A}}$ & & \\
\hline Albumin $(\mathrm{g} / \mathrm{dl})$ & $3.3 \pm 0.1$ & $3.2 \pm 0.1$ & $3.7 \pm 0.1$ & $3.9 \pm 0.3$ & & \\
\hline Total bilirubin (mg/dl) & $0.31 \pm 0.05$ & $0.25 \pm 0.03$ & $0.55 \pm 0.10$ & $0.24 \pm 0.03^{A}$ & & \\
\hline Lactate dehydrogenase $(\mathrm{U} / \mathrm{I})$ & $721 \pm 108$ & $564 \pm 97$ & $1,220 \pm 390$ & $1830 \pm 320$ & & \\
\hline Oxygen consumption $\left(\mathrm{ml} / \mathrm{h} / \mathrm{g}^{0.75}\right)$ & $10.33 \pm 0.36$ & $10.86 \pm 0.24$ & $8.25 \pm 0.24$ & $8.23 \pm 0.20$ & & \\
\hline RER & $0.699 \pm 0.006$ & $0.692 \pm 0.005$ & $0.730 \pm 0.007$ & $0.698 \pm 0.007^{A}$ & & \\
\hline
\end{tabular}

Female WT or A-ZIP/F-1 mice, 4-5 weeks old, were treated with rosiglitazone for 5 weeks. Female $o b / o b$ mice, 6 weeks old, were treated for 2 weeks. Animals were sacrificed in the fed state. Data are mean $\pm \operatorname{SEM}(n=6)$. ${ }^{A} P<0.05$ for differences within each genotype between control and rosiglitazone-treated mice. ${ }^{B} P<0.005$ for differences within each genotype between control and rosiglitazone-treated mice. 
the response to rosiglitazone treatment, we measured hepatic PPAR $\gamma$ mRNA levels. WT mice had levels of PPAR $\gamma$ mRNA near the limit of detection by Northern analysis. In contrast, in A-ZIP/F-1 mice, the PPAR $\gamma$ mRNA levels were increased (3- to 17-fold; quantitation of the increase is difficult owing to the low levels in the WT mice; Figure 4, e and f, and Figure 5). Rosiglitazone treatment had little effect on PPAR $\gamma$ mRNA levels in WT mice, but increased them 1.4-fold in A-ZIP/F-1 mice. Fat transplantation reversed the increase in the A-ZIP/F-1 mice. In skeletal muscle, Northern blots demonstrated barely detectable levels of PPAR $\gamma$ mRNA in A-ZIP/F-1 mice, FVB/N controls, and $o b / o b$ mice, which did not change upon rosiglitazone treatment (data not shown). Thus, there is a striking correlation between the hepatic PPAR $\gamma$ mRNA levels, the hepatic triglyceride content, and the response to rosiglitazone treatment. These data suggest that PPAR $\gamma$ levels are an important determinant of hepatic triglyceride content, presumably increasing lipogenesis and possibly fatty acid uptake.

The aP2 gene is transcribed predominantly in adipose and other PPAR $\gamma$-responsive tissues $(34,35)$. Hepatic aP2 mRNA levels were similar in A-ZIP/F-1 and control mice but were induced by rosiglitazone treatment only in the A-ZIP/F-1 mice (FVB/N controls, 100 and 59, vs. A-ZIP/F-1, 101 and 565; first value is untreated, second is rosiglitazone-treated; data are given in arbitrary units and are averages of two different pooled samples). These data indicate that hepatic PPAR $\gamma$ drives liver expression of aP2, a gene usually thought of as an adipose marker.

We also quantitated the liver mRNAs encoding transcription factors and metabolic enzymes involved in lipogenesis, $\beta$-oxidation, and glucose homeostasis. In A-ZIP/F-1 mice, the lipogenic genes, fatty acid synthase (FAS) and stearoyl-CoA desaturase-1 (SCD1), were both higher than in control mice (Figure 5). After 5 weeks of rosiglitazone treatment, SCD1 levels increased approximately 1.7-fold in both WT and A-ZIP/F-1 mice, whereas FAS levels did not change. Liver mRNA levels for SREBP1 were slightly elevated in A-ZIP/F-1 mice, but were not affected by rosiglitazone treatment.

To study the effect of rosiglitazone on $\beta$-oxidation genes, we measured PPAR $\alpha$, carnitine palmitoyl transferase-1 (CPT1), and acyl-CoA oxidase (AOX) mRNA levels (Figure 5). These mRNAs were all expressed at similar levels in control WT and A-ZIP/F-1 mice. Rosiglitazone treatment significantly increased mRNA levels only for AOX, which catalyzes the first step of $\beta$ oxidation. The AOX increase was less than that seen with treatment with PPAR $\alpha$ agonist treatment (24).

Phosphoenolpyruvate carboxykinase (PEPCK) mRNA levels indicate the level of gluconeogenesis. In A-ZIP/F-1 mice, the PEPCK mRNA levels were comparable to those of control mice. IRS-2 mRNA levels, which correlate with insulin signaling (36), were reduced by $80 \%$ in A-ZIP/F-1 mice and were slightly less reduced after rosiglitazone treatment.
In summary, the mRNA levels in untreated A-ZIP/F1 mice demonstrate increased expression of lipogenic enzymes, with no change in enzymes involved in $\beta$-oxidation. Rosiglitazone treatment caused a modest increase in AOX mRNA levels in A-ZIP/F-1, but not WT mice, consistent with an increase in $\beta$-oxidation.
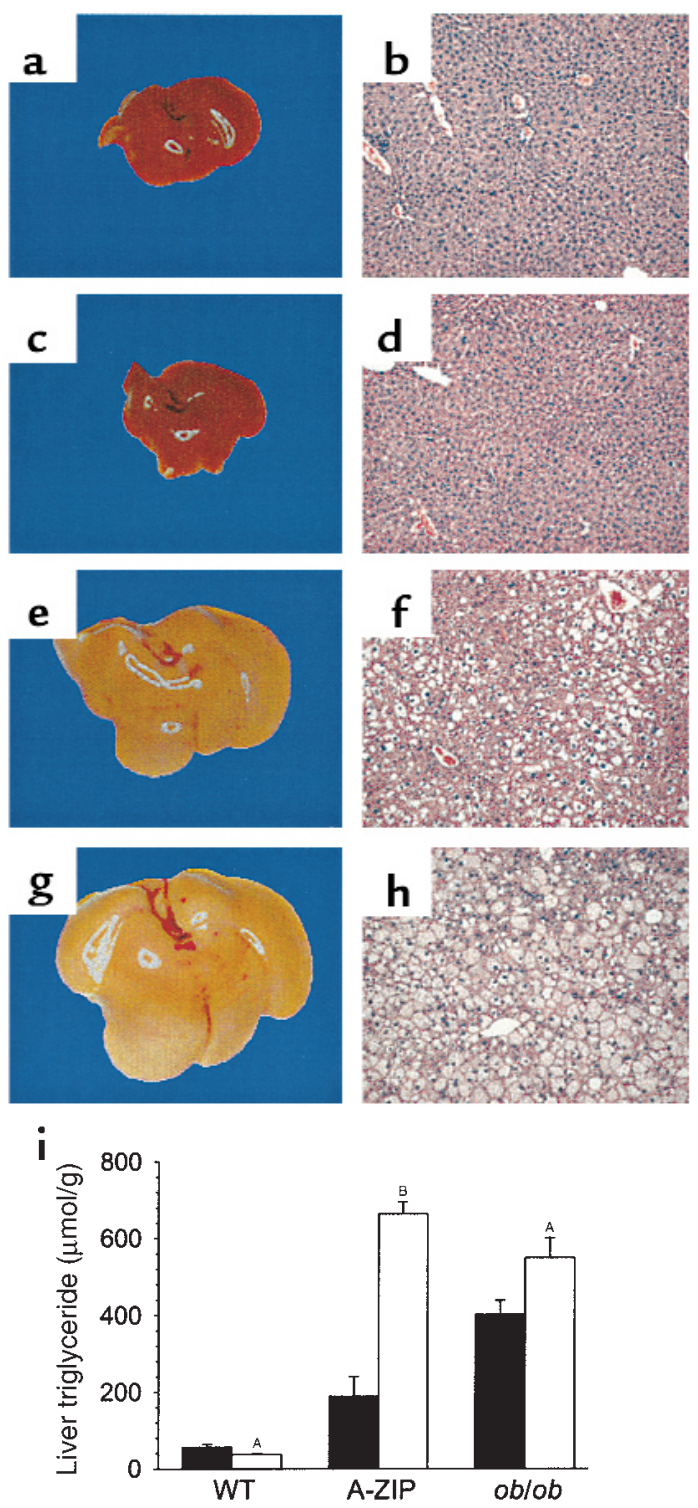

Figure 3

Rosiglitazone exacerbates hepatic steatosis. Livers from WT (a-d) and A-ZIP/F-1 (e-h) mice, either treated with rosiglitazone $(\mathbf{c}, \mathbf{d}, \mathbf{g}, \mathbf{h})$ or not $(\mathbf{a}, \mathbf{b}, \mathbf{e}, \mathbf{f})$ for 5 weeks, are shown at the same magnification. Hematoxylin and eosin-stained sections of the same livers (original magnification, $\times 100$ ) show steatosis of the control A-ZIP/F-1 mice, which worsens with rosiglitazone treatment. Liver triglyceride content (i) is shown for control (filled bars) and rosiglitazone-treated (open bars) mice (after 5 weeks of treatment for the A-ZIP/F-1 mice and 2 weeks for the $o b / o b$ mice). Data are mean $\pm \operatorname{SEM}(n=5-6) .{ }^{A} P<0.05$ for differences within each genotype between control and rosiglitazone-treated mice. ${ }^{\mathrm{B}} P<0.005$ for differences within each genotype between control and rosiglitazone-treated mice. 


\section{Discussion}

White adipose tissue is required for the insulin-sensitizing actions of TZDs. We show that rosiglitazone (or troglitazone) treatment of lipoatrophic A-ZIP/F-1 mice does not improve their hyperglycemia or hyperinsulinemia. This result is most consistent with the hypothesis that WAT is required for the antidiabetic effects of TZDs. The adipose site for TZD action is concordant with the observation that PPAR $\gamma$ is normally expressed at high levels in adipose tissue and not the other major insulin target tissues, muscle and liver. The effect of the TZDs in diabetics without lipoatrophy may be through the stimulation of adipocyte differentiation, generating more small adipocytes, which are more insulin sensitive. The TZDs may also increase insulin sensitivity by stimulating adipocyte glucose, fatty acid, and triglyceride uptake; reducing systemic levels; or altering release of adipose signaling molecules that affect insulin sensitivity in other tissues.

Fat transplantation did not confer rosiglitazoneresponsiveness to the $\mathrm{A}-\mathrm{ZIP} / \mathrm{F}-1$ mice. There are a number of possible explanations for this failure. First, the transplanted fat may have lowered the serum glucose and insulin too efficiently, such that a rosiglitazone effect was masked. Second, although fat implants vascularize and contain nerves, their function and regulation are not entirely normal (4), so the transplanted fat may not have been able to respond to TZDs. Third, the transplanted adipocytes may have enlarged to the point at which they were poorly responsive to TZDs. Finally, the transplanted WAT may have exhausted its proliferative potential. Thus the lack of restoration of the antidi- abetic effects of rosiglitazone by fat transplantation does not detract from our belief in the importance of adipose tissue in mediating the antidiabetic effects of TZDs.

Mice lacking PPAR $\gamma$ have been produced (37-39). Homozygous PPAR $\gamma$ disruption is embryonic lethal, but $P P A R \gamma^{t-}$ mice are viable. Surprisingly, when challenged with a high fat diet, $P P A R \gamma^{\dagger-}$ mice show increased insulin sensitivity relative to $P P A R \gamma^{t /+}$ controls $(38,40)$. A proposed explanation is that the $P P A R \gamma^{+/-}$adipose tissue is not as lipid laden and is thus more insulin-sensitive, possibly with a contribution from the paradoxically higher leptin levels (41).

The conclusion that WAT is required for the antidiabetic effects of TZDs contrasts with that reached by Burant et al. (22). Using the aP2/DTA mouse model of lipoatrophy, they concluded that troglitazone action is independent of adipose tissue. The aP2/DTA mouse has a normal amount of WAT at weaning, which begins to diminish by approximately 5 months of age, and by the time of troglitazone treatment (8-9 months), these mice showed an approximately $90 \%$ reduction in gonadal fat. The effectiveness of thiazolidinediones in aP2-nSREBP-1c lipoatrophic mice with intermediate levels of adipose tissue (42) has not been reported.

There are multiple reasons for the difference between our conclusions and those of Burant et al. The mice express different transgenes and have different genetic backgrounds. Also, the greater residual adipose tissue of the aP2/DTA mouse may enable improvement in the diabetes via direct adipose effects, just as troglitazone effectively treats the diabetes of patients with partial lipodystrophy (32).

\section{Figure 4}

Effect of rosiglitazone treatment on fat-transplanted A-ZIP/F-1 mice. WAT (400 mg) was transplanted into A-ZIP/F-1 mice at 5 weeks of age. Serum glucose (a) and insulin (b) were measured weekly (note that the insulin scale is logarithmic). Rosiglitazone (or control) treatment was begun 2 weeks after transplantation. Symbols are filled circles, sham-operated AZIP/F-1; open circles, sham-operated, rosiglitazone-treated A-ZIP/F-1; filled triangles, transplanted A-ZIP/F-1; open triangles, transplanted, rosiglitazone-treated A-ZIP/F-1; and filled squares, WT FVB/N. Hepatic weight (c), triglyceride content (d), and PPAR $\gamma$ mRNA (e, f) levels in control (filled bars) or rosiglitazonetreated (open bars) mice at 8 weeks after transplant. WAT transplants increased to 329 $\pm 59 \%$ and $398 \pm 87 \%$ of initial weight in the control and rosiglitazone-treated groups, respectively. mRNA levels are expressed as a percent of WT levels. Each lane of the Northern blot is from a different mouse. Data are mean $\pm \operatorname{SEM}(n=5-6)$. a

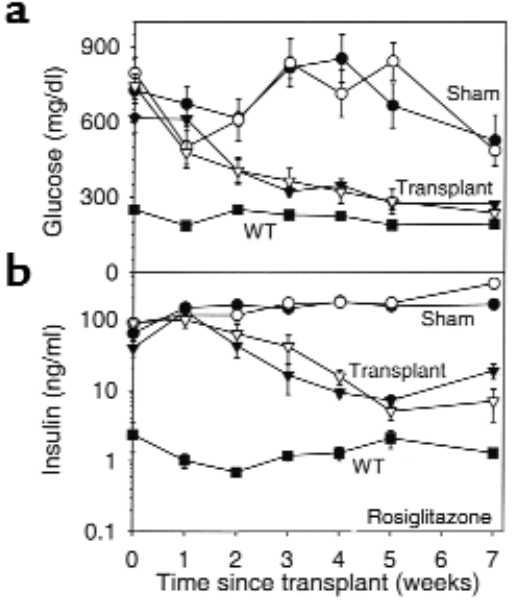

C
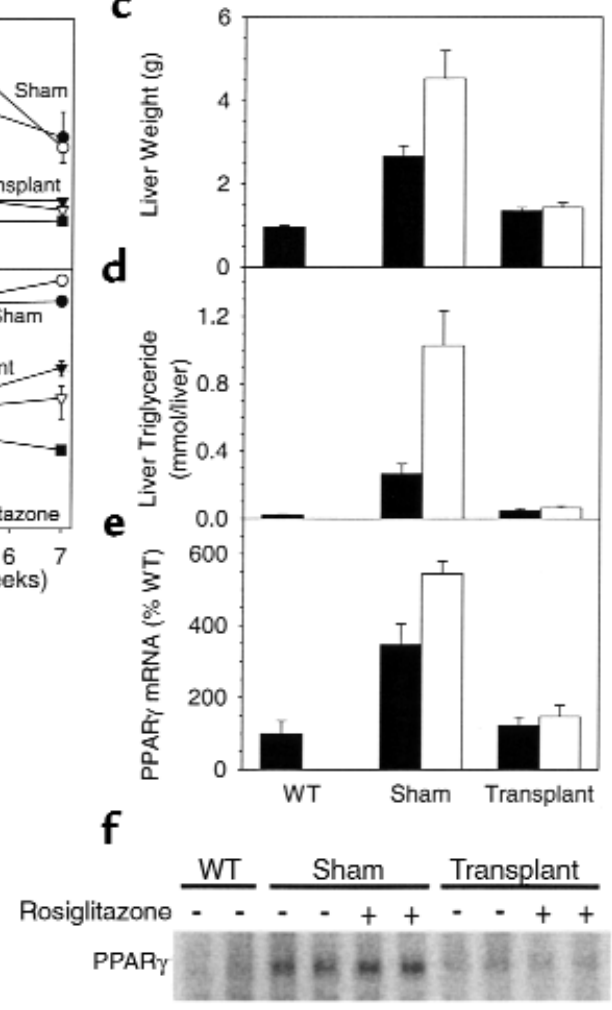
Rosiglitazone improves hypertriglyceridemia in A-ZIP/F-1 mice, independently of white adipose tissue. In contrast to its lack of antidiabetic effects, rosiglitazone lowered serum triglycerides and FFAs in A-ZIP/F-1 mice. Possible mechanisms are decreased dietary intake, decreased fatty acid production, increased $\beta$-oxidation, and redistribution away from the circulating compartment. Food intake was not decreased, indicating that triglyceride availability did not change. Although we have not measured fatty acid production directly, neither FAS nor SCD1 mRNA was decreased (and in fact these mRNAs were increased), indicating that decreased hepatic fatty acid synthesis is unlikely. In contrast, increased fat oxidation probably does contribute to the improvement in lipid levels, as hepatic AOX mRNA levels are increased and the elevated respiratory quotient was reversed by rosiglitazone treatment. We do not know which tissues contribute to the increased lipid oxidation in the AZIP/F-1 mice; muscle and liver are candidates. It is also possible that redistribution of circulating triglycerides into tissue occurs. For example, uptake of circulating lipids may contribute to the fatty liver.

TZD actions and PPAR $\gamma$ physiology in mice with normal compared with steatotic livers. PPAR $\gamma$ is normally expressed prominently in white adipose tissue, with much lower levels in liver and skeletal muscle $(12-14,17)$. The AZIP/F-1 mice have increased hepatic PPAR $\gamma$ mRNA levels. This increase in PPAR $\gamma$ may be a general property of steatotic livers, as it is present in $o b / o b$ mice (43), UCPDTA mice fed a high-fat diet (14), PPAR $\alpha$ knockout mice (44), and slightly in aP2/DTA mice (22). The mechanistic relationship between the steatosis and increased PPAR $\gamma$ mRNA levels is unclear. Do increased PPAR $\gamma$ levels cause increased lipogenesis and/or lipid uptake, leading to steatosis? Does hepatic steatosis per se lead to increased PPAR $\gamma$ levels? Or are both true? One possibility is that during increased energy availability (such as obesity or a high-fat diet), a circulating factor stimulates hepatic PPAR $\gamma$ transcription, turning on the lipogenic program and producing a fatty liver.

The very different responses to rosiglitazone treatment of A-ZIP/F-1 and $o b / o b$ mice (with PPAR $\gamma$-rich, fatty livers) compared with WT and fat-transplanted A-ZIP/F-1 mice (with low PPAR $\gamma$ and normal or near-normal liver lipid) highlight a fundamental difference between normal and lipoatrophic mice. In normal mice, there is little hepatic PPAR $\gamma$ and thus little direct hepatic effect of rosiglitazone treatment. The major rosiglitazone action on adipocytes provides more storage capacity for triglyceride, resulting in slightly lower hepatic (and other tissue) triglyceride levels. In contrast, in lipoatrophic mice, the steatotic liver is a major direct target for rosiglitazone action and becomes more lipogenic upon rosiglitazone treatment, further increasing liver lipid content.

Although both rosiglitazone and troglitazone failed to lower glucose and insulin, and both did reduce triglycerides, there were significant differences between these two TZDs. Notably, only rosiglitazone increased hepatic triglyceride content. It is possible that one or
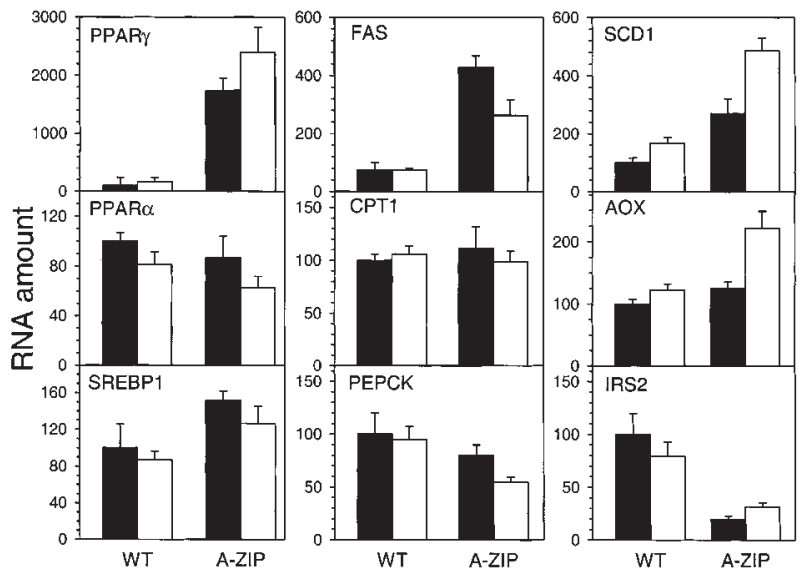

\section{Figure 5}

Liver mRNA levels. Results are for control (filled bars) and rosiglitazone-treated (open bars) mice after 5 weeks of treatment. Data are expressed as a percentage of WT control and are mean \pm SEM $(n=4-5)$. By two-way ANOVA, the genotype effect is significant $(P<0.05)$ for all except PPAR $\alpha$ and CPT1; the treatment effect is significant for SCD1 and AOX, and there is a significant genotype $x$ treatment interaction only for AOX.

both of these drugs have differential effects via PPAR $\gamma$ independent mechanisms (45). Another likely explanation is that troglitazone is a partial PPAR $\gamma$ agonist in certain assays, whereas rosiglitazone is a full agonist, giving the drugs overlapping but distinct effects on gene expression (46). Recent reports of PPAR $\gamma$ antagonist (47) and weak agonist (48) ligands hint at the future pharmacological diversity. In theory, an ideal PPAR $\gamma$ ligand would target adipose tissue, but not liver, and increase insulin sensitivity, but not lipogenesis. Generation of A-ZIP/F-1 mice with liver-specific disruption of PPAR $\gamma$ will test the contribution of hepatic PPAR $\gamma$ to generation of the fatty liver.

Obesity is a major risk factor for both diabetes and hepatic steatosis, so it is likely that both conditions will be present in a significant number of people. Given that the physiology of PPAR action (particularly PPAR $\alpha$ ) can be different between mice and humans, our results raise clinical questions that can only be answered with data from humans. Do people with steatotic livers have increased hepatic PPAR $\gamma$ levels? What about patients with type 2 diabetes? Do humans with hepatic steatosis respond differently to (certain) TZDs than do people with lean livers? We are not aware of studies measuring PPAR $\gamma$ levels in steatotic human livers. Nor do we know of reports specifically comparing the effects of TZDs in patients with fatty livers compared with controls. However, when a group of lipoatrophy patients was treated with troglitazone, both improved diabetes and a reduced liver volume (presumably due to reduced triglyceride content) were observed (32). The diabetes and liver volume improved even in the four patients with the most severe generalized lipoatrophy (E. Arioglu and S.I. Taylor, personal communication). One interpretation is that there are 
differences in physiology between humans and mice. Our results suggest that the antidiabetic actions of TZDs require adipose tissue. However, the absence of adipose tissue causes severe hepatic steatosis with high PPAR $\gamma$ levels. In this situation, rosiglitazone treatment worsened the steatosis, while lowering circulating triglycerides and increasing net fractional fatty acid oxidation. We postulate that the state of the liver determines the mouse's response to rosiglitazone treatment, with PPAR $\gamma$-rich fatty livers becoming even more lipidladen, whereas normal livers actually lose triglycerides.

\section{Acknowledgments}

We thank S. Taylor for stimulating discussions, M. Eckhaus for review of histology, J. Peters for plasmids, SmithKline Beecham Pharmaceuticals for rosiglitazone, and Parke Davis Research for troglitazone. C.J. Chou, P. Gorden, M. Haluzik, S. Taylor, and L. Weinstein provided helpful comments on the manuscript. Lily Chao is a Howard Hughes Medical Institute-NIH Research Scholar.

1. Moitra, J., et al. 1998. Life without white fat: a transgenic mouse. Genes Dev. 12:3168-3181.

2. Shimomura, I., Hammer, R.E., Ikemoto, S., Brown, M.S., and Goldstein, J.L. 1999. Leptin reverses insulin resistance and diabetes mellitus in mice with congenital lipodystrophy. Nature. 401:73-76.

3. Gavrilova, O., Marcus-Samuels, B., Leon, L.R., Vinson, C., and Reitman, M.L. 2000. Leptin and diabetes in lipoatrophic mice. Nature. 403:850; discussion $850-851$.

4. Gavrilova, O., et al. 2000. Surgical implantation of adipose tissue reverses diabetes in lipoatrophic mice. J. Clin. Invest. 105:271-278.

5. Kim, J.K., Gavrilova, O., Chen, Y., Reitman, M.L., and Shulman, G.I. 2000 Mechanism of insulin resistance in A-ZIP/F-1 fatless mice. J. Biol. Chem. 275:8456-8460.

6. Unger, R.H. 1995. Lipotoxicity in the pathogenesis of obesity-dependent NIDDM. Genetic and clinical implications. Diabetes. 44:863-870.

7. Shimabukuro, M., et al. 1997. Direct antidiabetic effect of leptin through triglyceride depletion of tissues. Proc. Natl. Acad. Sci. USA. 94:4637-4641.

8. Foyt, H.L., Ghazzi, M.N., Hanley, R.M., Saltiel, A.R., and Whitcomb, R.W. 2000. Thiazolidinediones. In Diabetes mellitus: a fundamental and clinical text. 2nd edition. D. LeRoith, S.I. Taylor, and J.M. Olefsky, editors. Lippincott Williams \& Wilkins. Philadelphia, Pennsylvania, USA. 788-797.

9. Olefsky, J.M. 2000. Treatment of insulin resistance with peroxisome proliferator-activated receptor $\gamma$ agonists. J. Clin. Invest. 106:467-472.

10. Oakes, N.D., et al. 1994. A new antidiabetic agent, BRL 49653, reduces lipid availability and improves insulin action and glucoregulation in the rat. Diabetes. 43:1203-1210.

11. Lehmann, J.M., et al. 1995. An antidiabetic thiazolidinedione is a high affinity ligand for peroxisome proliferator-activated receptor gamma (PPAR gamma). J. Biol. Chem. 270:12953-12956.

12. Zhu, Y., Alvares, K., Huang, Q., Rao, M.S., and Reddy, J.K. 1993. Cloning of a new member of the peroxisome proliferator-activated receptor gene family from mouse liver. J. Biol. Chem. 268:26817-26820.

13. Tontonoz, P., Hu, E., Graves, R.A., Budavari, A.I., and Spiegelman, B.M. 1994. $\mathrm{mPPAR}$ gamma 2: tissue-specific regulator of an adipocyte enhancer. Genes Dev. 8:1224-1234

14. Vidal-Puig, A., et al. 1996. Regulation of PPAR gamma gene expression by nutrition and obesity in rodents. J. Clin. Invest. 97:2553-2561.

15. Michael, L.F., Lazar, M.A., and Mendelson, C.R. 1997. Peroxisome proliferator-activated receptor gamma 1 expression is induced during cyclic adenosine monophosphate-stimulated differentiation of alveolar type II pneumonocytes. Endocrinology. 138:3695-3703.

16. DuBois, R.N., et al. 1998. The nuclear eicosanoid receptor, PPARgamma, is aberrantly expressed in colonic cancers. Carcinogenesis. 19:49-53.

17. Kruszynska, Y.T., et al. 1998. Skeletal muscle peroxisome proliferator-activated receptor-gamma expression in obesity and non-insulin-dependent diabetes mellitus. J. Clin. Invest. 101:543-548.

18. Kersten, S., Desvergne, B., and Wahli, W. 2000. Roles of PPARs in health and disease. Nature. 405:421-424.

19. Maggs, D.G., et al. 1998. Metabolic effects of troglitazone monotherapy in type 2 diabetes mellitus. A randomized, double-blind, placebo-controlled trial. Ann. Intern. Med. 128:176-185.

20. Tontonoz, P., Hu, E., and Spiegelman, B.M. 1994. Stimulation of adipogenesis in fibroblasts by PPAR gamma 2, a lipid-activated transcription factor. Cell. 79:1147-1156.

21. Okuno, A., et al. 1998. Troglitazone increases the number of small adipocytes without the change of white adipose tissue mass in obese Zucker rats. J. Clin. Invest. 101:1354-1361.

22. Burant, C.F., et al. 1997. Troglitazone action is independent of adipose tissue. J. Clin. Invest. 100:2900-2908.

23. Gavrilova, O., et al. 1999. Torpor in mice is induced by both leptin-dependent and -independent mechanisms. Proc. Natl. Acad. Sci. USA. 96:14623-14628

24. Lee, S.S., et al. 1995. Targeted disruption of the alpha isoform of the peroxisome proliferator-activated receptor gene in mice results in abolishment of the pleiotropic effects of peroxisome proliferators. Mol. Cell. Biol. 15:3012-3022

25. Yoo-Warren, H., et al. 1981. Identification of a DNA clone to phosphoenolpyruvate carboxykinase (GTP) from rat cytosol. Alterations in phosphoenolpyruvate carboxykinase RNA levels detectable by hybridization. J. Biol. Chem. 256:10224-10227.

26. Shimano, H., et al. 1996. Overproduction of cholesterol and fatty acids causes massive liver enlargement in transgenic mice expressing truncated SREBP-1a. J. Clin. Invest. 98:1575-1584.

27. Cantello, B.C., et al. 1994. [[omega-(Heterocyclylamino)alkoxy]benzyl]-2,4thiazolidinediones as potent antihyperglycemic agents. J. Med. Chem. 37:3977-3985

28. Ghazzi, M.N., et al. 1997. Cardiac and glycemic benefits of troglitazone treatment in NIDDM. The Troglitazone Study Group. Diabetes. 46:433-439.

29. Antonucci, T., Whitcomb, R., McLain, R., Lockwood, D., and Norris, R.M. 1997. Impaired glucose tolerance is normalized by treatment with the thiazolidinedione troglitazone. Diabetes Care. 20:188-193.

30. Chaput, E., Saladin, R., Silvestre, M., and Edgar, A.D. 2000. Fenofibrate and rosiglitazone lower serum triglycerides with opposing effects on body weight. Biochem. Biophys. Res. Commun. 271:445-450.

31. McLean, J.A., and Tobin, G. 1987. Animal and human calorimetry. Cambridge University Press. Cambridge, United Kingdom. 338 pp.

32. Arioglu, E., et al. 2000. Efficacy and safety of troglitazone in the treatment of lipodystrophy syndromes. Ann. Intern. Med. 133:263-274.

33. Gavrilova, O., Marcus-Samuels, B., and Reitman, M.L. 2000. Lack of responses to a beta3 adrenergic agonist in lipoatrophic A-ZIP/F-1 mice. Diabetes. 49:1910-1916.

34. Zezulak, K.M., and Green, H. 1985. Specificity of gene expression in adipocytes. Mol. Cell. Biol. 5:419-421.

35. Pelton, P.D., Zhou, L., Demarest, K.T., and Burris, T.P. 1999. PPARgamma activation induces the expression of the adipocyte fatty acid binding protein gene in human monocytes. Biochem. Biophys. Res. Commun. 261:456-458.

36. Shimomura, I., et al. 2000. Decreased IRS-2 and increased SREBP-1c lead to mixed insulin resistance and sensitivity in livers of lipodystrophic and ob/ob mice. Mol. Cell. 6:77-86.

37. Barak, Y., et al. 1999. PPAR gamma is required for placental, cardiac, and adipose tissue development. Mol. Cell. 4:585-595.

38. Kubota, N., et al. 1999. PPAR gamma mediates high-fat diet-induced adipocyte hypertrophy and insulin resistance. Mol. Cell. 4:597-609.

39. Rosen, E.D., et al. 1999. PPAR gamma is required for the differentiation of adipose tissue in vivo and in vitro. Mol. Cell. 4:611-617.

40. Miles, P.D., Barak, Y., He, W., Evans, R.M., and Olefsky, J.M. 2000. Improved insulin-sensitivity in mice heterozygous for PPAR-gamma deficiency. J. Clin. Invest. 105:287-292

41. Kadowaki, T. 2000. Insights into insulin resistance and type 2 diabetes from knockout mouse models. J. Clin. Invest. 106:459-465.

42. Shimomura, I., et al. 1998. Insulin resistance and diabetes mellitus in transgenic mice expressing nuclear SREBP-1c in adipose tissue: model for congenital generalized lipodystrophy. Genes Dev. 12:3182-3194.

43. Edvardsson, U., et al. 1999. Rosiglitazone (BRL49653), a PPARgamma-selective agonist, causes peroxisome proliferator-like liver effects in obese mice. J. Lipid Res. 40:1177-1184

44. Costet, P., et al. 1998. Peroxisome proliferator-activated receptor alpha-isoform deficiency leads to progressive dyslipidemia with sexually dimorphic obesity and steatosis. J. Biol. Chem. 273:29577-29585.

45. Jones, S.A., et al. 2000. The pregnane $X$ receptor: a promiscuous xenobiotic receptor that has diverged during evolution. Mol. Endocrinol. 14:27-39.

46. Camp, H.S., et al. 2000. Differential activation of peroxisome proliferatoractivated receptor- gamma by troglitazone and rosiglitazone. Diabetes. 49:539-547.

47. Mukherjee, R., et al. 2000. A selective peroxisome proliferator-activated receptor-gamma modulator blocks adipocyte differentiation but stimulates glucose uptake in 3T3-L1 adipocytes. Mol. Endocrinol. 14:1425-1433.

48. Oberfield, J.L., et al. 1999. A peroxisome proliferator-activated receptor gamma ligand inhibits adipocyte differentiation. Proc. Natl. Acad. Sci. USA. 96:6102-6106 